\title{
Factorization and domination of positive Banach-Saks operators
}

\author{
by \\ Julio Flores and Pedro Tradacete (Madrid)
}

\begin{abstract}
It is proved that every positive Banach-Saks operator $T: E \rightarrow F$ between Banach lattices $E$ and $F$ factors through a Banach lattice with the Banach-Saks property, provided that $F$ has order continuous norm. By means of an example we show that this order continuity condition cannot be removed. In addition, some domination results, in the Dodds-Fremlin sense, are obtained for the class of Banach-Saks operators.
\end{abstract}

1. Introduction. Factorization properties of operators between Banach spaces have been widely studied. It is well known that every weakly compact operator between Banach spaces factors through a reflexive Banach space [6]. In contrast, M. Talagrand showed that, in general, a weakly compact operator between Banach lattices cannot be expected to factor through a reflexive Banach lattice [20]. However, C. D. Aliprantis and O. Burkinshaw proved that this can be done under quite general assumptions [1].

Regarding the class of Banach-Saks operators between Banach spaces, Beauzamy provided in [5] an analogous factorization result. Namely, every Banach-Saks operator factors through a Banach space with the BanachSaks property. In this note we want to consider the analogous question in the Banach lattice context. In particular, we will prove that every positive Banach-Saks operator from a Banach lattice to an order continuous Banach lattice factors through a Banach lattice with the Banach-Saks property (see Theorem 2.1). In addition, we show that Talagrand's example [20] can be used to prove that the order continuity hypothesis cannot be removed (see Example 1).

2000 Mathematics Subject Classification: 46B42, 47B65, 47B07.

Key words and phrases: Banach-Saks property, Banach lattice, positive operator, factorization.

Both authors were partially supported by grants MEC MTM2005-00082 and Santander/Complutense PR34/07-15837. Second author was partially supported by grant MEC AP-2004-4841. 
In the second part of this note we give a domination result for the class of Banach-Saks operators (Corollary 3.3) which improves the results previously obtained in [12]. Recall that the domination problem for a class $\mathcal{C}$ of operators acting between Banach lattices is stated as follows:

Problem 1. Let $0 \leq R \leq T: E \rightarrow F$ be two positive operators. Assume that $T$ belongs to the class $\mathcal{C}$. What conditions on $E$ and $F$ ensure that $R$ belongs to $\mathcal{C}$ ?

For positive endomorphisms on a Banach lattice the power problem is closely related:

Problem 2. Let $0 \leq R \leq T: E \rightarrow E$, and $T \in \mathcal{C}$. What is the smallest $n \in \mathbb{N}$ such that $R^{n} \in \mathcal{C}$ ?

Corollary 3.2 provides an answer to this question for the class of BanachSaks operators.

Power and domination problems have been studied for other classes of operators in [3], [4], [7], [14], [21], and more recently in [11] and [15].

We refer to the books [16], [17] and [2] for unexplained terms and notation.

Acknowledgements. The authors would like to thank Professor N. J. Kalton for very helpful conversations.

\section{Factorization of operators through Banach lattices with the} Banach-Saks property. Recall that an operator between Banach spaces $T: X \rightarrow Y$ is Banach-Saks if every bounded sequence $\left(x_{n}\right)$ in $X$ has a subsequence such that $\left(T x_{n_{k}}\right)$ is Cesàro convergent, that is, the sequence of arithmetic means $\left(N^{-1} \sum_{k=1}^{N} T\left(x_{n_{k}}\right)\right)_{N}$ is convergent in the norm of $Y$. A Banach space is said to have the Banach-Saks property if the identity operator is Banach-Saks. We say that a Banach space $X$ has the weak BanachSaks property if every weakly null sequence in $X$ has a Cesàro convergent subsequence.

For convenience, we will say that a subset $S$ of a Banach space $X$ is a Banach-Saks set if for every sequence $\left(x_{n}\right)$ in $S$, there exists a subsequence $\left(x_{n_{k}}\right)$ that is Cesàro convergent. Clearly, an operator $T: X \rightarrow Y$ is BanachSaks if and only if $T\left(B_{X}\right)$ is a Banach-Saks set, where $B_{X}$ is the closed unit ball of $X$.

The main result of this note is the following:

Theorem 2.1. Let $E$ and $F$ be Banach lattices and $T: E \rightarrow F a$ positive Banach-Saks operator. If $F$ is order continuous, then there exist a 
Banach lattice $H$ with the Banach-Saks property and operators $T_{1}: E \rightarrow H$, $T_{2}: H \rightarrow F$ such that the following factorization diagram holds:

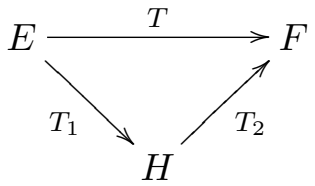

Before the proof, we need to collect some definitions and facts. Recall that an order continuous Banach lattice $E$ with a weak unit can be considered as an (in general non-closed) order ideal of $L_{1}(\Omega, \Sigma, \mu)$ for a certain probability space $(\Omega, \Sigma, \mu)$, such that the natural inclusion $E \hookrightarrow L_{1}(\Omega, \Sigma, \mu)$ is continuous with norm smaller than one [17, Prop. 1.b.14]. Recall also that in an order continuous Banach lattice every ideal is complemented by a positive projection [17, Prop. 1.b].

Let $E$ be a Banach function space with order continuous norm defined over a finite measure space $(\Omega, \Sigma, \mu)$. Recall that a bounded subset $A \subset E$ is equi-integrable if for every $\varepsilon>0$ there exists $\delta>0$ such that $\left\|f \chi_{B}\right\|_{E}<\varepsilon$ for every $B \in \Sigma$ with $\mu(B)<\delta$ and every $f \in A$. The following result is known (see [10, Lemma 3.3] for a proof).

Lemma 2.2. Let $E$ be a Banach lattice with order continuous norm and a weak unit, and hence representable as an order ideal in $L_{1}(\Omega, \Sigma, \mu)$ for some probability space $(\Omega, \Sigma, \mu)$. A norm bounded sequence $\left(g_{n}\right)$ in $E$ is convergent if and only if it is equi-integrable and \|\|$_{1}$-convergent.

Proof of Theorem 2.1. First of all, we claim that the solid hull of $T\left(B_{E}\right)$ is a Banach-Saks set.

Indeed, since $T$ is Banach-Saks, $T\left(B_{E}\right)$ is a Banach-Saks set. Let $W$ denote the solid hull of $T\left(B_{E}\right)$. Take an arbitrary sequence $\left(z_{k}\right)$ in $W$. This sequence satisfies $\left|z_{k}\right| \leq\left|T x_{k}\right|$ for certain $x_{k} \in B_{E}$. For every $k$, take $r_{k}=$ $\left|x_{k}\right|$ in $B_{E}$; then

$$
\left|z_{k}\right| \leq T x_{k} \mid \leq T r_{k}
$$

Since $T$ is Banach-Saks, there exist $g \in F$ and some subsequence $\left(k_{s}\right)$ such that

$$
\frac{1}{m} \sum_{s=1}^{m} \operatorname{Tr}_{k_{s}} \rightarrow g
$$

in the norm of $F$. In fact, by [8] we can assume that the same property holds for every subsequence of $\left(k_{s}\right)$.

Take $M$, the closed ideal of $F$ generated by $x=g+\sum_{s=1}^{\infty} z_{k_{s}} / 2^{s}$. According to the remarks above, denote by $i: M \hookrightarrow L_{1}(\Omega, \Sigma, \mu)$ the continuous 
inclusion of $M$ as an order continuous Banach lattice with weak unit into some $L_{1}(\Omega, \Sigma, \mu)$, and let $P: F \rightarrow M$ be a positive projection onto $M$.

Clearly, the composition

$$
i P T: E \rightarrow L_{1}(\Omega, \Sigma, \mu)
$$

is a Banach-Saks operator. In particular, $i P T\left(B_{E}\right)$ is relatively weakly compact and so is its own convex solid hull, as $L_{1}(\Omega, \Sigma, \mu)$ is a band in its bidual [2, Theorem 4.39]. Hence, there exists $f \in L_{1}(\Omega, \Sigma, \mu)$ such that $i\left(z_{k_{s}}\right) \rightarrow f$ in the weak topology of $L_{1}(\Omega, \Sigma, \mu)$. Since $L_{1}(\Omega, \Sigma, \mu)$ has the weak BanachSaks property [19], passing to a further subsequence we can assume that

$$
\frac{1}{m} \sum_{s=1}^{m} i\left(z_{k_{s}}\right) \rightarrow f
$$

in the norm of $L_{1}(\Omega, \Sigma, \mu)$.

Notice that

$$
\left|\frac{1}{m} \sum_{s=1}^{m} z_{k_{s}}\right| \leq \frac{1}{m} \sum_{s=1}^{m} \operatorname{Tr}_{k_{s}},
$$

and $m^{-1} \sum_{s=1}^{m} \operatorname{Tr}_{k_{s}} \rightarrow g$ in the norm of $F$. It follows that $\left(m^{-1} \sum_{s=1}^{m} z_{k_{s}}\right)_{m}$ is equi-integrable in $F$, and therefore convergent in $F$ by the previous lines and Lemma 2.2. Thus, $W$ is a Banach-Saks set as claimed.

Consider now $F_{0}$, the completion of the space $\{z \in F: \exists \lambda<\infty, z \in \lambda W\}$ under the norm induced by the Minkowski functional of $W$. Since $W$ is solid and convex, the space $F_{0}$ is in fact a Banach lattice. Hence, so is $\left(F_{0}, F\right)_{\theta, p}$ $(0<\theta<1,1<p<\infty)$, the space obtained by Lions-Peetre interpolation of $F_{0}$ and $F\left[17,2\right.$.g]. Moreover, by $\left[5\right.$, Thm. 2], $\left(F_{0}, F\right)_{\theta, p}$ has the Banach-Saks property.

Finally, since $T\left(B_{E}\right) \subset W$, the operator $T: E \rightarrow F_{0}$ is bounded. Thus, by the interpolation theorem [17, Prop. 2.g.15], $T$ is bounded from $E$ to $\left(F_{0}, F\right)_{\theta, p}$. Let $T_{1}: E \rightarrow\left(F_{0}, F\right)_{\theta, p}$ denote this operator. Since $W \subset B_{F}$, we also see that the inclusion $i:\left(F_{0}, F\right)_{\theta, p} \hookrightarrow F$ is bounded. Therefore, we have the factorization

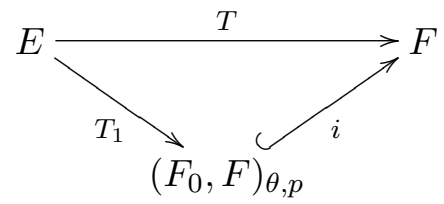

Take $H=\left(F_{0}, F\right)_{\theta, p}$ and $T_{2}=i$ to conclude the proof.

Corollary 2.3. Let $E$ be a Banach lattice. If $0 \leq T: E \rightarrow E$ is Banach-Saks, then $T^{2}$ factors through a Banach lattice with the BanachSaks property. 
Proof. Since $c_{0}$ does not have the Banach-Saks property, $T$ cannot be an isomorphism on any subspace of $E$ isomorphic to $c_{0}$. Hence, by [13, Thm. I.2], there exist an order continuous Banach lattice $F$ and positive operators $R$ and $S$ such that

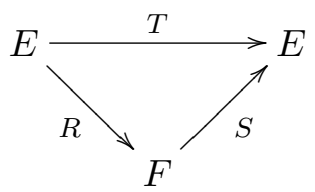

Therefore, since $F$ is order continuous, Theorem 2.1 implies that $R T: E \rightarrow$ $F$ factors through a Banach lattice $H$ with the Banach-Saks property. Hence, $T^{2}=S R T$ also factors through $H$ as claimed.

Note that, in general, every Banach-Saks operator between Banach spaces factors through a Banach space with the Banach-Saks property [5, Thm. 1]. However, if the operator acts between Banach lattices it is not true in general that the space obtained in such a factorization has to be a lattice. To see this we will benefit from the well-known example provided by Talagrand [20] of a positive weakly compact operator between Banach lattices which fails to factor through any reflexive Banach lattice. Since Banach-Saks property implies reflexivity it suffices to prove that Talagrand's operator is in fact Banach-Saks. Thus, Theorem 2.1 (and Corollary 2.3) turns out to be optimal in a sense.

Let us briefly recall for the reader's convenience the construction of Talagrand's operator. First, let

$L=\left\{h: \mathbb{N} \cup\{\infty\} \rightarrow\{0,1\}: \exists p \leq i_{1}<\cdots<i_{p}, h(i)=0\right.$ for $\left.i \neq i_{1}, \ldots, i_{p}\right\}$.

Then $L \subset C(\mathbb{N} \cup\{\infty\})$ is weakly compact. For every $l \geq 1$ consider the map

$$
\theta_{l}: L^{l} \rightarrow C\left((\mathbb{N} \cup\{\infty\})^{l}\right),
$$

defined as $\theta_{l}\left(h_{1}, \ldots, h_{l}\right)\left(n_{1}, \ldots, n_{l}\right)=1$ if the number of indices $i$ for which $h_{i}\left(n_{i}\right)=1$ is even, and $\theta_{l}\left(h_{1}, \ldots, h_{l}\right)\left(n_{1}, \ldots, n_{l}\right)=0$ otherwise. Notice that since $\theta_{l}$ is continuous for the topology of pointwise convergence in $C((\mathbb{N} \cup$ $\left.\{\infty\})^{l}\right)$, the set $K_{l}=\theta_{l}\left(L^{l}\right)$ is weakly compact.

Let $M$ be the Aleksandrov compactification of the discrete sum of the sets $(\mathbb{N} \cup\{\infty\})^{l}$. Each $K_{l}$ can be considered as a subset of $C(M)$ by extending the functions of $K_{l}$ to zero outside $(\mathbb{N} \cup\{\infty\})^{l}$. Let $K=\bigcup_{l} K_{l}$. By construction, $K$ consists of $\{0,1\}$-valued functions, so $K$ is contained in the positive cone of $C(M)$.

Lemma 2.4. The closed convex hull of $K, \overline{\mathrm{co}} K$, is a Banach-Saks set.

Proof. Indeed, take $\left(y_{n}\right)$ arbitrarily in $\overline{c o} K$. We want to show that there is a subsequence of $\left(y_{n}\right)$ whose arithmetic means are convergent. For each 
$n \in \mathbb{N}$ write

$$
y_{n}=\sum_{j=1}^{\infty} \lambda_{n, j} w_{n, j},
$$

where $\sum_{j=1}^{\infty} \lambda_{n, j}=1, \lambda_{n, j} \geq 0$, and $w_{n, j}$ belongs to $W_{j}=\overline{\mathrm{co}} K_{j}$.

Passing to a subsequence of $\left(y_{n}\right)$, we can assume that for all $j \in \mathbb{N}$, there exists $\lambda_{j}$ such that $\lambda_{n, j} \stackrel{n}{\rightarrow} \lambda_{j}$, with $\sum_{j=1}^{\infty} \lambda_{j}=1$. Let

$$
y_{n}^{\prime}=\sum_{j=1}^{\infty} \lambda_{j} w_{n, j} \quad \text { and } \quad e_{n}=y_{n}-y_{n}^{\prime} .
$$

Since $K_{j}$ is weakly compact, so is $W_{j}$; hence, passing to a further subsequence we can assume that for each $j \in \mathbb{N}$ there is some $z_{j} \in W_{j}$ such that $w_{n, j} \stackrel{n}{\rightarrow} z_{j}$ weakly. Note that for each $j \in \mathbb{N}, W_{j}$ is weakly compact in $C\left((\mathbb{N} \cup\{\infty\})^{j}\right)$, which is isomorphic to $c_{0}$. Since $c_{0}$ has the weak Banach-Saks property [9], we deduce that $W_{j}$ is a Banach-Saks set. Hence, using [8] and a diagonal process, we can extract a subsequence $\left(n_{i}\right)$ such that for each $j \in \mathbb{N}$ there exists $f_{j}: \mathbb{N} \rightarrow \mathbb{R}$ satisfying

$$
\left\|\sum_{i=1}^{k} w_{n_{i}, j}-k z_{j}\right\| \leq f_{j}(k)
$$

and $f_{j}(k) / k \rightarrow 0$ as $k \rightarrow \infty$. Since the $W_{j}$ are disjointly supported on $M$ we get

$$
\left\|\sum_{i=1}^{k} y_{n_{i}}^{\prime}-k \sum_{j=1}^{\infty} \lambda_{j} z_{j}\right\|=\left\|\sum_{j=1}^{\infty} \lambda_{j}\left(\sum_{i=1}^{k} w_{n_{i}, j}-k z_{j}\right)\right\| \leq \max _{j} \lambda_{j} f_{j}(k),
$$

which implies

$$
\frac{1}{k} \sum_{i=1}^{k} y_{n_{i}}^{\prime} \stackrel{k}{\rightarrow} \sum_{j=1}^{\infty} \lambda_{j} z_{j}
$$

in the norm of $C(M)$. Hence, $\left(y_{n_{i}}^{\prime}\right)$ (and every subsequence of it) has convergent arithmetic means.

A gliding hump argument shows that $\left(e_{n}\right)$ has a subsequence equivalent to the unit vector basis of $c_{0}$. Indeed, taking an appropriate subsequence we can assume that $\lambda_{n, j} \rightarrow \lambda_{j}$ fast enough, so that the following construction can be carried out. First, set $n_{1}=1$ and let $j_{1}$ be such that

$$
\left\|\sum_{j=j_{1}}^{\infty}\left(\lambda_{n_{1}, j}-\lambda_{j}\right) w_{n_{1}, j}\right\|<\frac{1}{2} \text {. }
$$


Next, take $n_{2}$ such that

$$
\left\|\sum_{j=1}^{j_{1}}\left(\lambda_{n_{2}, j}-\lambda_{j}\right) w_{n_{2}, j}\right\|<\frac{1}{2^{3}},
$$

and then choose $j_{2}$ such that

$$
\left\|\sum_{j=j_{2}}^{\infty}\left(\lambda_{n_{2}, j}-\lambda_{j}\right) w_{n_{2}, j}\right\|<\frac{1}{2^{3}} .
$$

In this way, we construct inductively a pair of sequences $\left(n_{k}\right)$ and $\left(j_{k}\right)$ such that

$$
\sum_{k=1}^{\infty}\left\|e_{n_{k}}-\sum_{j=j_{k-1}}^{j_{k}-1}\left(\lambda_{n_{k}, j}-\lambda_{j}\right) w_{n_{k}, j}\right\| \leq 1 .
$$

Thus, $\left(e_{n_{k}}\right)$ is equivalent to $\left(\sum_{j=j_{k-1}}^{j_{k}}\left(\lambda_{n_{k}, j}-\lambda_{j}\right) w_{n_{k}, j}\right)_{k}$, which is a disjoint sequence in $C(M)$ equivalent to the unit vector basis of $c_{0}$.

Finally, note that every subsequence of the unit vector basis of $c_{0}$ has convergent arithmetic means. Therefore, both $\left(e_{n_{k}}\right)$ and $\left(y_{n_{k}}^{\prime}\right)$ have subsequences with the same property. This implies that the same is true for some subsequence of $\left(y_{n}\right)$, and the proof is finished.

EXAMPLE 1. There exists a positive operator $U: \ell_{1} \rightarrow C[0,1]$ which is Banach-Saks but fails to factor through a Banach lattice with the BanachSaks property.

Proof. Note that $K$ can be seen as a subset of $C[0,1]$ by taking a positive embedding of $C(M)$ into $C([0,1])$ such that its image is complemented. Take a dense sequence $\left(x_{n}\right)$ in $K$ and consider the operator

$$
U: \ell_{1} \rightarrow C([0,1]), \quad\left(a_{n}\right)_{n=1}^{\infty} \mapsto \sum_{n=1}^{\infty} a_{n} x_{n} .
$$

Clearly, $U$ is positive. Moreover, $U\left(B_{\ell_{1}}\right)=$ co $K$ is a Banach-Saks set by Lemma 2.4, and therefore the operator $U$ is Banach-Saks.

By [20, Thm. A] the operator $U: \ell_{1} \rightarrow C([0,1])$ does not factor through any reflexive Banach lattice. Since every space with the Banach-Saks property is reflexive, the proof is finished.

This shows that the hypotheses in Theorem 2.1 are necessary. Moreover, if we consider the operator $\widetilde{U}: \ell_{1} \oplus C([0,1]) \rightarrow \ell_{1} \oplus C([0,1])$ given by $\widetilde{U}(x, y)=(0, U(x))$, then one notices that Corollary 2.3 cannot be improved.

3. Domination of Banach-Saks operators. In this section we look at the problem of domination for Banach-Saks operators on Banach lattices. Recall that an operator $T: E \rightarrow X$ from a Banach lattice $E$ to a 
Banach space $X$ is order weakly compact if $T$ maps order intervals to weakly precompact sets [2, p. 318]. Observe that given $0 \leq R_{1} \leq T_{1}: E_{1} \rightarrow E_{2}$ and $0 \leq R_{2} \leq T_{2}: E_{2} \rightarrow E_{3}$ with $T_{1}$ Banach-Saks and $T_{2}$ order weakly compact, the proof of Theorem 2.1 can be adapted to obtain the factorization

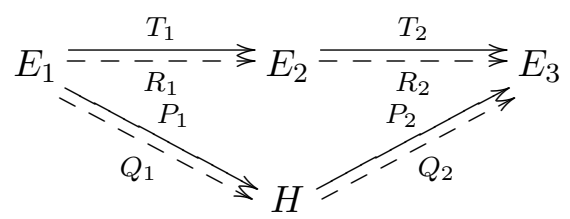

where $H$ is a Banach lattice with the Banach-Saks property, $0 \leq Q_{1} \leq P_{1}$ and $0 \leq Q_{2} \leq P_{2}$. From this a domination result for Banach-Saks operators is easily obtained. However, we provide an alternative proof of this fact which does not depend on interpolation. This is the content of the following result which improves some previous work in [12].

Theorem 3.1. Let $E_{1}, E_{2}$ and $E_{3}$ be Banach lattices and $0 \leq R_{i} \leq T_{i}$ : $E_{i} \rightarrow E_{i+1}$ be positive operators for $i=1$, 2. If $T_{1}$ is a Banach-Saks operator and $T_{2}$ is order weakly compact, then the composition $R_{2} R_{1}$ is a Banach-Saks operator.

Proof. Since $T_{2}$ is order weakly compact, by [13, Thm. I.2] we have the factorization

$$
E_{1} \frac{T_{1}}{--R_{1}^{-} \rightarrow} E_{2} \frac{T_{2}}{---\bar{R}_{2}--\gg} E_{3}
$$

where $F$ is an order continuous Banach lattice and $0 \leq Q \leq P$.

Take an arbitrary sequence $\left(x_{n}\right)$ in $B_{E_{1}}$ and the closed ideal generated by $\left(\left|\phi T_{1}\left(x_{n}\right)\right|\right)$ in $F \hookrightarrow L_{1}(\Omega, \Sigma, \mu)$ as above.

Since $T_{1}$ is Banach-Saks and

$$
\left|\phi R_{1} x_{n_{k}}\right| \leq \phi T_{1}\left|x_{n_{k}}\right|,
$$

there exists a subsequence $\left(n_{k}\right)$ such that the arithmetic means

$$
\frac{1}{m} \sum_{k=1}^{m} \phi T_{1}\left|x_{n_{k}}\right|
$$

converge to some $x \in F$. Then $\left(c_{m}=m^{-1} \sum_{k=1}^{m} \phi R_{1} x_{n_{k}}\right)_{m}$ is an equiintegrable sequence in $F$ (and the same is true for any subsequence of $\left(n_{k}\right)$ by [18]). 
Since $\phi T_{1}$ is weakly compact, Gantmacher's theorem implies in particular that the adjoint $\left(\phi T_{1}\right)^{*}$ is order weakly compact, so we get a factorization for $\phi T_{1}$ and $\phi R_{1}$ through a Banach lattice $G$ such that both $G$ and $G^{*}$ are order continuous [13, Prop. I.4 and Thm. I.6]:

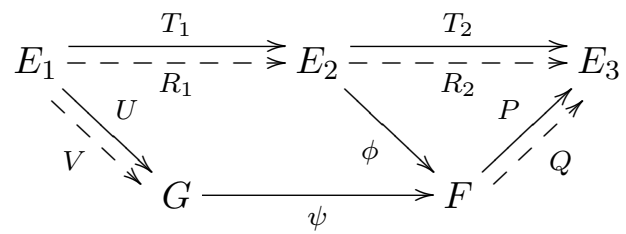

By passing to some subsequence, [2, Theorem 4.25] shows that $\left(\phi R_{1} x_{n_{k}}\right)$ is weakly Cauchy, hence weakly convergent in $L_{1}(\Omega, \Sigma, \mu)$. Now, by [19], $\left(\phi R_{1} x_{n_{k}}\right)$ has a subsequence whose arithmetic means converge in the norm of $L_{1}(\Omega, \Sigma, \mu)$ to some function $f \in L_{1}(\Omega, \Sigma, \mu)$. However, since

$$
\left|\frac{1}{m} \sum_{k=1}^{m} \phi R_{1} x_{n_{k}}\right| \leq \frac{1}{m} \sum_{k=1}^{m} \phi T_{1}\left|x_{n_{k}}\right|,
$$

and

$$
\frac{1}{m} \sum_{k=1}^{m} \phi T_{1}\left|x_{n_{k}}\right| \rightarrow x
$$

for some $x \in F$, we must have $|f| \leq x$, which implies that $f \in F$. Therefore the sequence of arithmetic means, $\left(c_{m}\right)$, must be convergent in the norm of $F$ (see Lemma 2.2). This implies that $\phi R_{1}$ and consequently $R_{2} R_{1}$ are Banach-Saks operators.

Corollary 3.2. Let $E$ be a Banach lattice and $0 \leq R \leq T: E \rightarrow E$ be positive operators. If $T$ is Banach-Saks, then so is $R^{2}$.

Proof. Since $T$ is Banach-Saks, it is also weakly compact [18], and in particular order weakly compact. Theorem 3.1 yields the result.

Note that in [12, Ex. 2.9] it was shown that there exist operators

$$
0 \leq R \leq T: \ell_{1} \rightarrow \ell_{\infty}
$$

such that $T$ is Banach-Saks, but $R$ is not. This shows that Corollary 3.2 is sharp; indeed, consider the operators $0 \leq \widetilde{R} \leq \widetilde{T}: \ell_{1} \oplus \ell_{\infty} \rightarrow \ell_{1} \oplus \ell_{\infty}$ defined by

$$
\widetilde{R}=\left(\begin{array}{cc}
0 & 0 \\
R & 0
\end{array}\right), \quad \widetilde{T}=\left(\begin{array}{cc}
0 & 0 \\
T & 0
\end{array}\right) .
$$

Clearly $\widetilde{T}$ is Banach-Saks, but $\widetilde{R}$ is not. Notice that $\widetilde{R}^{2}=0$.

We also have the following improvement upon [12, Thm. 1.1]. 
Corollary 3.3. Let $E$ and $F$ be Banach lattices such that $F$ is order continuous. If $0 \leq R \leq T: E \rightarrow F$ with $T$ Banach-Saks, then $R$ is also a Banach-Saks operator.

Proof. Use Theorem 3.1 and the fact that order intervals in an order continuous Banach lattice are weakly compact [17, p. 28].

The following question remains open: Can order continuity on $F$ be replaced with order continuity on $E^{*}$ in Corollary 3.3?

\section{References}

[1] C. D. Aliprantis and O. Burkinshaw, Factoring compact and weakly compact operators through reflexive Banach lattices, Trans. Amer. Math. Soc. 283 (1984), 369-381.

[2] —, - Positive Operators, Springer, Dordrecht, 2006.

[3] - - - Positive compact operators on Banach lattices, Math. Z. 174 (1980), 289-298.

[4] - - - On weakly compact operators on Banach lattices, Proc. Amer. Math. Soc. 83 (1981), 573-578.

[5] B. Beauzamy, Propriété de Banach-Saks, Studia Math. 66 (1980), 227-235.

[6] W. J. Davis, T. Figiel, W. B. Johnson and A. Pełczyński, Factoring weakly compact operators, J. Funct. Anal. 17 (1974), 311-327.

[7] P. G. Dodds and D. H. Fremlin, Compact operators in Banach lattices, Israel J. Math. 34 (1979), 287-320.

[8] P. Erdôs and M. Magidor, A note on regular methods of summability and the Banach-Saks property, Proc. Amer. Math. Soc. 59 (1976), 232-234.

[9] N. R. Farnum, The Banach-Saks theorem in $C(S)$, Canad. J. Math. 26 (1974), 91-97.

[10] J. Flores and F. L. Hernández, Domination by positive strictly singular operators, J. London Math. Soc. (2) 66 (2002), 433-452.

[11] J. Flores, F. L. Hernández and P. Tradacete, Powers of operators dominated by strictly singular operators, Quart. J. Math., in press (doi: 10.1093/qmath/ham050).

[12] J. Flores and C. Ruiz, Domination by positive Banach-Saks operators, Studia Math. 173 (2006), 185-192.

[13] N. Ghoussoub and W. B. Johnson, Factoring operators through Banach lattices not containing $C(0,1)$, Math. Z. 194 (1987), 153-171.

[14] N. Kalton and P. Saab, Ideal properties of regular operators between Banach lattices, Illinois J. Math. 29 (1985), 382-400.

[15] C. C. A. Labuschagne, A Dodds-Fremlin property for Asplund and Radon-Nikodým operators, Positivity 10 (2006), 391-407.

[16] J. Lindenstrauss and L. Tzafriri, Classical Banach Spaces I: Sequence Spaces, Springer, 1977.

[17] -, 一, Classical Banach Spaces II: Function Spaces, Springer, 1979.

[18] T. Nishiura and D. Waterman, Reflexivity and summability, Studia Math. 23 (1963), $53-57$.

[19] W. Szlenk, Sur les suites faiblement convergentes dans l'espace L, ibid. 25 (1965), $337-341$.

[20] M. Talagrand, Some weakly compact operators between Banach lattices do not factor through reflexive Banach lattices, Proc. Amer. Math. Soc. 96 (1986), 95-102. 
[21] A. W. Wickstead, Extremal structure of cones of operators, Quart. J. Math. Oxford Ser. (2) 32 (1981), 239-253.

Department of Applied Mathematics, Escet Universidad Rey Juan Carlos 28933, Móstoles, Madrid, Spain

E-mail: julio.flores@urjc.es
Departamento de Análisis Matemático Facultad de Ciencias Matemáticas Universidad Complutense de Madrid 28040, Madrid, Spain E-mail: tradacete@mat.ucm.es

Received February 23, 2008

Revised version June 29, 2008 